\title{
The Principles of Fuel Oil Engines-I
}

\author{
The Chemical and Physical Basis of Their Operation
}

By C. F. Hirshfeld

IT is not the intention of this paper to consider the various real liquid fuel engines nor to discuss the weak and strong points of such engines as have already bee constructed. I have taken a different method of attack and, going back to first principles, have attempted to analyze the different phenomena which enter and to draw conclusions therefrom.

Doubtless much that I say will prove to be already known to many, but I hope that the orderly arrangement and the co-relating of such knowledge may prove worth while. Personal experience has shown me that this is the only way in which the many apparently

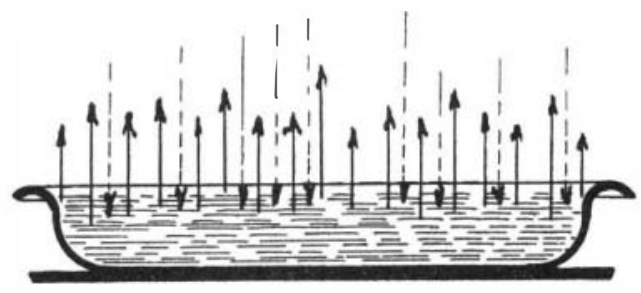

Fig. 1a.-Evaporation and recondensation.

contradictory phenomena met in liquid fuel engines can be properly appreciated.

We will first review certain simple physical phe nomena which may seem to have little connection with our subject, but it will be discovered later that they are all-important.

There is considerable misunderstanding of the phe nomena known as evaporation, vaporization, and boiling, largely because of a loose use of the terms. It is first necessary to clear up this part of the field and for this purpose we will start with Fig. $1 a$.

It is a matter of common experience that wate placed in an open vessel, such as that shown in Fig. 10 and left exposed to the atmosphere, will gradually disappear. We explain this by saying that the wate evaporates.

Actually, if we take the kinetic view of matter, the molecules of the liquid water are in rapid motion and the surface under conditions which permit it to penetrate it will do so and pass off into the space above. The full arrows in the figure are supposed to show how the molecules leave the surface and gradually dissipate in the space above, that is, how evaporation occurs.

If any water molecules, traveling in the space above the liquid surface, happen to strike that surface under conditions which will permit them to penetrate they will do so and thus become part of the liquid. Such action is what we commonly call condensation and is illustrated in the figure by the dotted arrows.

In general, under such conditions as we have a sumed, the molecules leaving the liquid will greatly outnumber those entering and therefore the water will gradually disappear or evaporate. Observe, however, that evaporation and condensation really go on at the silme time, and it is the relative ralue of the two phe

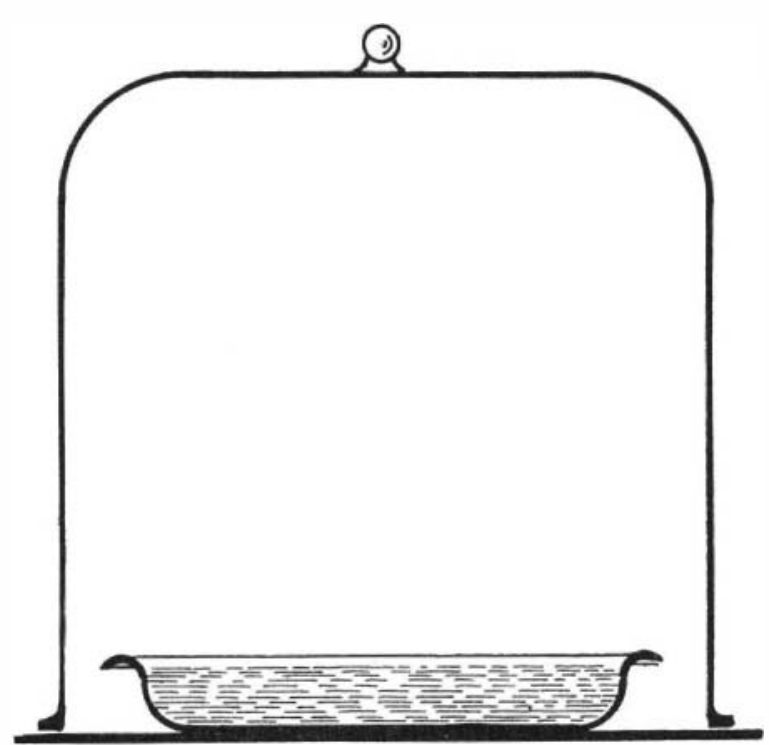

Fig. 1b.-Liquid and vapor in equilibrium.

nomena which determines whether the liquid present increases or decreases in quantity.

Taking one step more, experience shows us that the higher the temperature of the liquid axposed the more

- Paper read before the American Soclety of Agricultura bugineers, and published in the Gas Reviero. rapidly will it evaporate. 'Ihis simply means that the liquid molecules are moving faster at the higher temperature, more of them arrive at and penetrate the liquid surface in a given time, and hence more escape. If the space above the liquid be also at a high temperature, the chances of these molecules getting far from the liquid surface are great; if the temperature above be low, the chances for a large number returning are increased.

If the atmosphere above the liquid is moving across the liquid surface (a wind) the molecules leaving will, in general, be carried away before any of them have had a chance to return to the liquid and evaporation will be increased.

Let us now take the arrangement shown in Fig. $1 b$. This represents the same vessel of water with a bell jar placed orer it so as to inclose the space above the liquid. It is obvious that if we have enough water to start with, it will only be a matter of time before just as many molecules are returning to the liquid as are leaving it in a given time. That is, eventually the space above the surface will contain so many molecules of water that condensation will equal evaporation, and we would say in our approximate way that evaporation had ceased.

When this sort of equilibrium is reached we say that the space is saturated with the vapor of water, which really means that the space is filled with the saturated vapor of water.

Suppose, now, that we try an experiment with this apparatus. We will first maintain the whole thing at

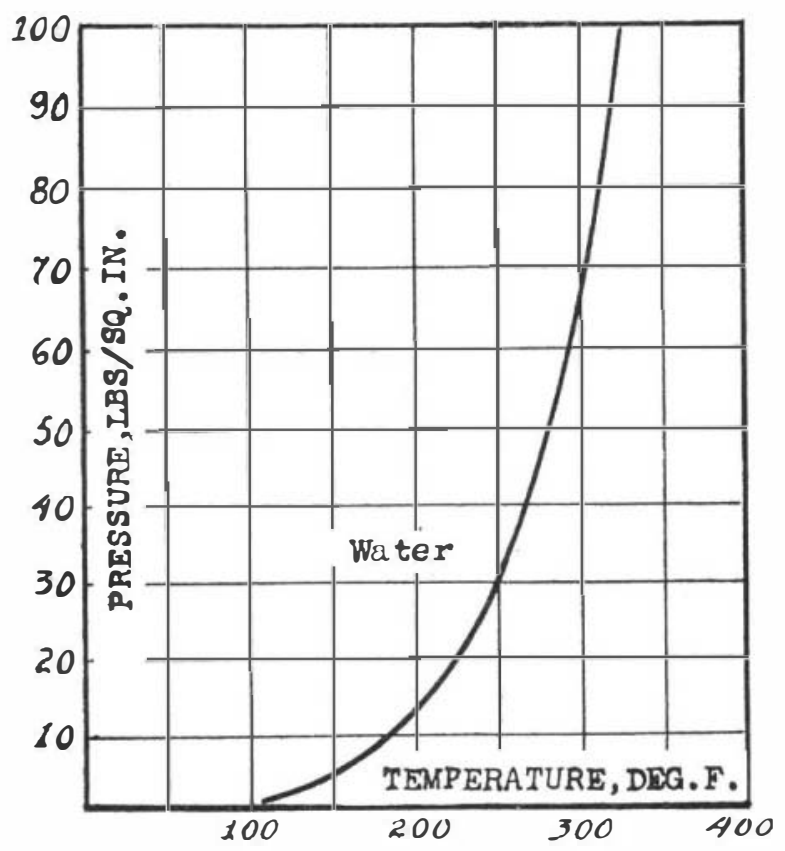

Fig. 1c. - Vapor-pressure curve of water.

some definite temperature and observe the level at tained by the liquid after equilibrium is reached. We will then maintain the whole apparatus at successively higher temperatures and observe the levels attained in each case. Our observations would show that the higher the temperature the lower the level of the water in the vessel when equilibrium is reached or, the higher above the linud when saturated conditions are reached.

But we know that the more molecules we pack into given space the greater is the pressure exerted by those molecules since more of them must strike th walls in a given period of time. It follows directly that the saturated vapor will exert greater pressure the higher its temperature.

This is a matter of common knowledge among engi neers who know that steam pressure $i$ e the pressure of saturated water vapor, increases with the temperature. This is shown graphically in Fig. $1 c$ which wa plotted from the data given in an ordinary steam table. While we are accustomed to speak of steam pressure at different temperatures what we really mean are the pressures of saturated water vapor at those temperatures or, in the words of the physicist, the vapor pressures of water.

It would, of course, make no difference in our reasoning if we substituted some other liquid for the water we have been discussing. We might, for instance, have used absolute ethyl alcohol, liquid hexane, liquid benzene, or any other material which is a liquid unde the conditions of our experiment Had we used such liquids as those mentioned we should have obtained results similar to those shown graphically in Fig. 1d. These curves were plotted from the vapor tables of the various liquids in question just as the previous curve was plotted from the steam table.

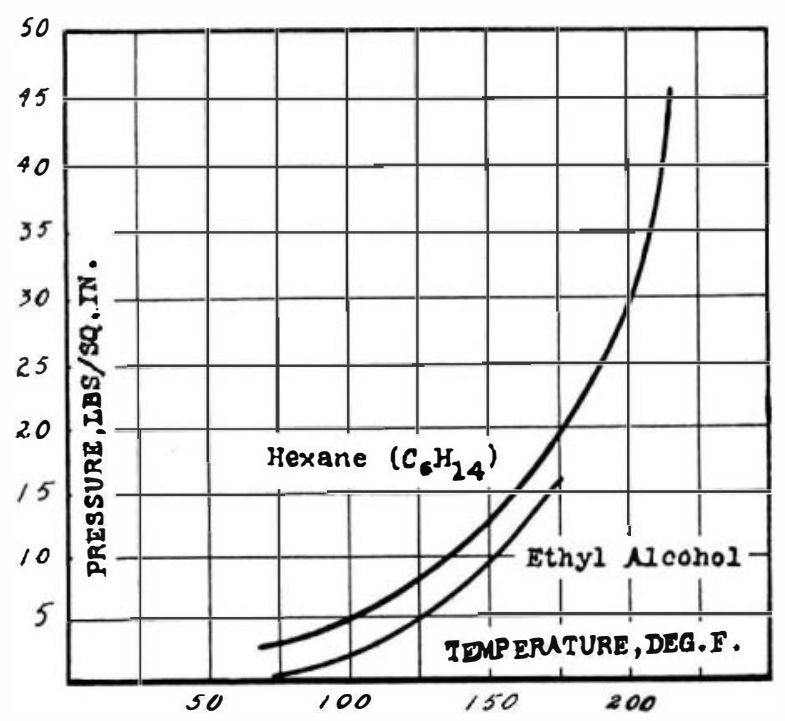

Fig. 1d.-Vapor-pressure curves of hexane and alcohol.

Assume now that we vary our experiment by introducing a pump which will circulate the water, as shown in Fig. 1e, so that we maintain a shower of drops falling into the liquid through the space above it. It is obvious that we expose much more surface of liquid than in the previous arrangement and therefore more molecules will escape in a given time. Hence we will fill the space more rapidly and reach equilibrium conditions more quickly. After equilibrium is attained, condensation on the surface of the drops and of the liquid in the vessel must equal the evaporation from those surfaces.

It is also obvious that the smaller we make the drops that is, the finer we spray, the more quickly will we reach equilibrium conditions. If we could spray so finely as to introduce single, separated molecules instead of drops of finite size, equilibrium would be attained almost instantaneously if we could pump fast enough.

But before we had reduced the drops to any such small dimensions we should have met another phenomenon. We should have discovered that the small drops did not immediately descend to the liquid in the vessel but remained floating in the form of fog. Under such conditions we could get a space saturated with the vapor and also filled with floating drops of the liquid. Such conditions are often described by saying that the space is supersaturated, but this is a very loose use of the word.

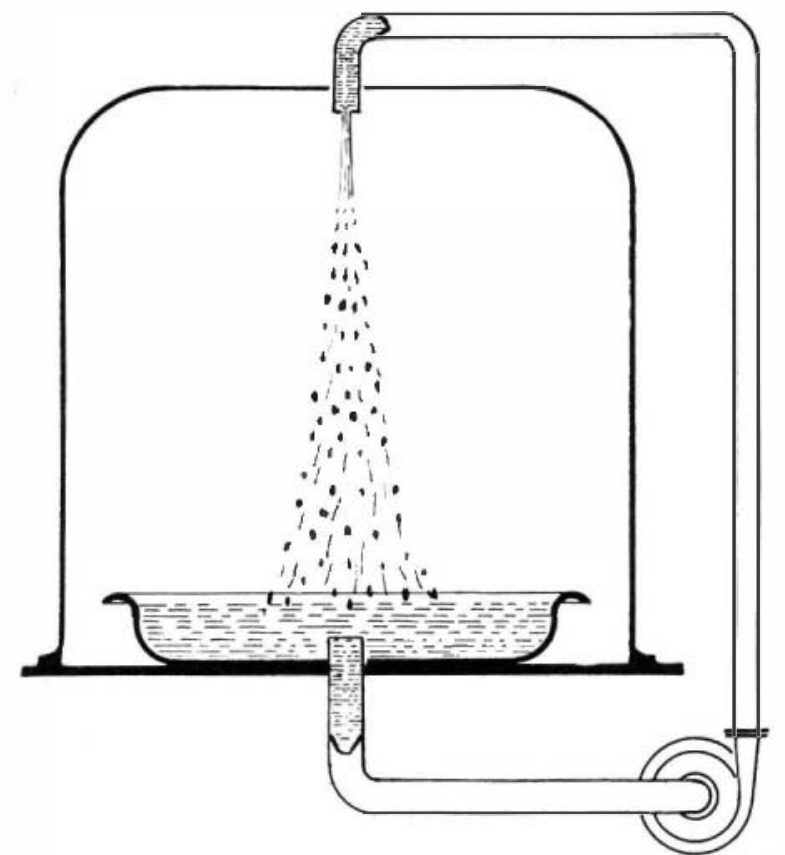

Fig. 1e.-A spray hastens establishment of equilibrium.

If we heated the space thus supersaturated the number of molecules required to saturate it would increase and consequently our finite drops would gradually evaporate and disappear. On the other hand, if we lowered the temperature some of the vapor molecules 
would separate out, that is condense, upon the liquid surfaces. Some would enter the surface in the vessel, but more would enter the surfaces of the drops in the space above. If the temperature were lowered to sufficient extent the drops could thus be made so heavy that they would no longer float, but would descend in a rain exactly like the rain which descends upon the earth.

Thus far we have said nothing about air within the bell jar though in any real case, had we performed the experiment as indicated, there would have been air present. We said nothing about it for the simple reason that its presence or absence makes no difference in the phenomena we have been discussing. We need consider temperature, and temperature only, for it is sider temperature, and temperature only, for it is
temperature alone which determines the number of temperature alone which determines the number of
molecules of any substance required to saturate a given space, and it is temperature alone which determines the pressure which those molecules (the vapor) are going to exert.

If air, or any similar neutral gas, happens to be present it will exert a pressure on the walls which bound the space, but, so far as the vapor is concerned, it might as well be absent. The vapor will exert the same pressure on the walls whether the air is there or not. The pressure recorded by the walls, however, will always be the combined pressure of air and vapor, that is, the sum of the two which are therefore called the partial pressures.

We can sum this up very simply by saying that so far as the vapor molecules and their motions are concerned the presence of the air molecules has no effect. It should, however, be observed that if two sets of molecules occupy the same space they must eventuall acquire the same temperature, and that this may in some cases lead to apparent contradictions of the prev ous statements.

There is one more fact about evaporation which should be noted. If we consider once more the apparatus in Fig. $1 b$ it is easy to prove that when we first put the bell jar over the vessel the rate at which the liquid surface is lowered by evaporation will be grea est and that it must decrease until it becomes zero when the saturated condition is reached. To prove this it is merely necessary to note that when the experiment starts there will be no molecules returning (assumin absolutely dry air) and that the number returning must grow greater until the saturated condition is attained

summing up what has preceded, we have discovered 1. Evaporation and condensation occur simultaneously

2. The apparent effect is merely the net result.
3. Increase of temperature of liquid and motion of the atmosphere will increase net evaporation.

4. A space is saturated with vapor when it contains so many molecules of that vapor that evaporation and ondensation are equal.

5. The number of molecules required to saturat ncreases with temperature, so high temperature space holds more weight than low temperature space.

6. This gives increasing pressure with increasing temperature, that is, vapor pressure increases with tem perat ure.

7. A space may hold more than the amount uired to saturate it if the excess exists as fog.

8. These phenomena are independent of any mateial which is inert with respect to the vapor under conderation.

9. The pressure of vapor in conflned space is dependent only on temperature if it is saturated, but pressure registered by walls is total or sum of all pressures within space.

10. Net evaporation is most rapid when space is arthest from saturated condition, less rapid the neare aturation is approached.

Let us now consider boiling in order to discover how it is related to evaporation. In the cases already considered we found that the presence of air had no effect The molecules of water might therefore be pictured as penetrating the spaces between the molecules of the air just as though the latter were not present. We increase the pressure of the saturated vapor, that is the vapor pressure.

Let us now imagine the vessel shown in Fig. $1 a$ to contain water and to be exposed to the air as before. The pressure on the surface of this liquid is undoubt edly that of the atmosphere which we will assume as the standard 14.7 pounds for convenience. Experience shows us that if we raise the temperature of the water to 212 deg. Fahr. what we call boiling will occur; that is, bubbles of vapor will form within the liquid, pass out, and disappear into the space above.

If you consult the steam tables or the curve shown in Fig. $1 c$ you will discover that the pressure of saturated water vapor at $212 \mathrm{deg}$. Fahr. is 14.7 pounds, that is, it is equal to that of the atmosphere under the as sumed conditions. It is evident, therefore, that what we call boiling or ebullition occurs when the temperature of the liquid has the value at which its vapor exerts a pressure equal to that upon the surface of the liquid.

As a matter of fact, the temperature of the molecules forming any particular bubble must be slightly highe than this in order that they may be able to push away the water as they form a bubble under the superincumpressure plus the head of water under which they density with respect to the surrounding water; it is literally pushed out into the atmosphere by the surrounding water. As soon as it enters the air it begins to break up, that is, the individual molecules each go their own way or diffusion occurs.

Boiling or ebullition is, then, merely a very rapid form of evaloration which occurs when the vapor pressure equals that on the liquid surface. Under these conditions molecules can push themselves out in groups instead of having to thread their way singly between the molecules of the superincumbent gas.

Suppose now that we change things slightly and produce a rain of drons of liquid at boiling temperature. Each drop is in such a condition that all of its surface can immediately flash into vapor and, if we assume a sufficient heat supply a vailable, there is no reason why the entire drop should not become a vapor and diffuse into the surrounding atmosphere. Such action would, of course, cease when we attained a saturated atmosphere since condensation would then be as rapid as raporization. In other words, we have merely devised a means of expediting evaporation beyond what was possible with previous arrangements.

The same result can be achieved in another way. We might devise a means of suspending small drops of liquid in air and then heat both the air and the drops. Lvaporation would result, and if we heated rapidly enough we might attain boiling temperature and get the same phenomena as before. If we heated slowly we might attain saturated conditions before boiling temperature was reached.

We have now considered processes of various linds which show that what we call ebullition or boiling is merely. a sort of limiting case of evaporation.

In all of the preceding we have entirely omitted any discussion of heat supply, assuming conveniently that such heat as might be required would be forthcoming. Actually, it requires very large amounts of heat to produce the evaporative results we have been considering. In the case of water the quantity is in the neighborhood of 1,000 British thermal units per pound; in the case of gasoline it is about 100 British thermal units, and in the case of alcohol (ethyl) in the neighborhood of 400 British thermal units.

If this heat is not supplied from some external source it will be taken from the vaporizing liquid itself anl from the air and apparatus.

(T॰ be continued.)

\section{The Uses of Artificial Insulating Materials in the Construction of Electrical Apparatus}

\author{
By H. Passarant
}

THE four most essential properties of an in sulating material may be stated as follows: (1) It must possess great mechanical toughness, so that if made in a suitable shape it can resist the must be capable to some extent of resisting the effects of heat, so that an accidental rise of temperature is not accompanied by any serious disintegration. (3) It must be capable of resistin the action of fire to such an extent that it is the action of fire to such an extent that it is stand the action of an arc; after such exposur to flame, it should not be found to have lost it insulating or mechanical properties. (4) It should to a sufficient extent, be able to resist the effects of moisture or chemical action, at any rate to such an extent as would be likely to be necessary in actual working. A commission has lately been engaged in investigating the properties of insulators, and it may be of interest to discuss som of the points which have, arisen in connection with that work.

If a piece of "Tenacit" of the kind that wa made a few years ago is held in the Bunsen flame the flame soon becomes colored and the material appears to catch fire after a short time; but as soon as the flame is removed, the tenacit ceases to burn. With a piece, exactly similar, but of the latest manufacture, these effects take place the latest manufacture, these effects take place
much more slowly, and it is extremely difficult to inflame the material at all; after removal of the flame the piece is found to be practically uninjured. If a plate of tenacit 10 millimeters
thick is taken, and some thermit is placed on it *Abstract of a paper read before the Eleltrotechnische Vorein
and published in The Electrician. and inflamed, causing a temperature of $2,500 \mathrm{deg}$. Cent. or 3,000 deg. Cent., a lightly burnt spot
will be noticed; but if the burnt surface is scratched away it will be found that the burning is only superficial, and that the rest of the material is quite uninjured. The electric arc must be taken into account as a disturbing influence in considering insulating materials, for it may be liable to be exposed to such an accident at any time. If the different materials are tested by exposing them to an are of 10 amperes at 220 volts between carbon electrodes, the arc being gradually drawn out to its greatest length, very different results are given by different materials. Thus marble tends to extinguish the arc; the electrodes can only be drawn out on the surface of the marble to a length of about 2 centimeters, when the are is extinguished. Slate is easily fused by the arc without becoming a conductor. The arc cannot be drawn out to a greater length than 4 centimeters. Inflammable materials are naturally set on fire, and conductive surfaces are therefore formed by the carbonization of the material, and the arc can therefore be drawn out to considerable lengths. Many substances that cannot be inflamed by an ordinary burner become inflammable in the are and burn with a sooty flame; and in this case the electrodes can be drawn out to a distance of about 12 centimeters. Tenacit of the latest type cannot be set on fire by the arc, and even after a length of time there is no soot formed. It only becomes conductive when fused and heated to a bright red heat, but the are can then only be maintained over slightly greater lengths than in air. A really good insulating material differs from porcelain and substances such as those used in tho Nernst lamp, which become conductors at high temperatures and help to maintain the arc instead of suppressing it. With respect to chemical inactivity, it is not possible to lay down very stringent conis able to withsenerally sufficient if the substance including snow, ice, and the light of the sun Such . Such a condition is not difficult to fulfil. It is rather
different from an order which was lately received and executed by the Allgemeine Elektrizitäts Gesellschaft for a vessel made of insulating material to hold electrolytic chlorine; the said vessel was delivered and has been working satisfactorily for a long time. The following experiment will help to give some idea of the toughness of tenacit. Suppose a plate of cast iron 3 millimeters thick to be placed on a layer of sand, and also a similar plate of tenacit. If now a steel ball weighing $1 / 2$ killigramme is allowed to fall on them from a height of about 1 meter, the tenacit plate will be broken or cracked, whereas the cast-iron one will remain uninjured. Suppose the tenacit plate to be replaced by another, having the same weight as the cast-iron one, and the experiment to be continued. It will then be found that the castiron will be shattered when the ball falls from a height of about 1.5 meters, whereas this will not take place with the tenacit until a height of about 2 meters is allowed for the fall. Naturally this proves nothing as to the relative strengths of the two materials, but it may help to give some sort of rough idea as to the toughness of the present manufacture.

These artificial insulating materials are used for many purposes, principally perbaps for small pieces of apparatus used in an ordinary house, or in 